\title{
Erratum to: Low-Temperature Transport in Metals without Inversion Centre
}

\author{
V. P. Mineev ${ }^{a, b}$ \\ ${ }^{a}$ Université Grenoble Alpes, CEA, IRIG, PHELIQS, Grenoble, F-38000 France \\ ${ }^{b}$ Landau Institute for Theoretical Physics, Chernogolovka, 142432 Russia \\ e-mail: vladimir.mineev@cea.fr \\ Received May 20, 2020
}

DOI: $10.1134 /$ S1063776120310015

Formula (32) given in the article is incorrect. The correct expression should read as follows:

$$
\operatorname{Im}\left(V_{ \pm i} V_{ \pm j}^{*}\right) E_{j}=\frac{1}{2} \hat{\gamma}\left(\frac{\partial \hat{\gamma}}{\partial k_{i}} \times \frac{\partial \hat{\gamma}}{\partial k_{j}}\right) E_{j},
$$

and in two-dimensional case is obviously equal to zero. In three-dimensional case, this expression is an odd function of $\mathbf{k}$, hence, the last term in equations (31) and (38) turns zero when integrated. Thus, the statement made in the article about the presence in the collisionless regime of a term in the current that is specific of a three-dimensional metal without center of inversion is incorrect.

The original article can be found online at

https://doi.org/10.1134/S1063776119100078 